\title{
EchoGéo
}

47| 2019

Nouvelles géographies de la collecte

\section{Déchets, mauvaises herbes et plantes sauvages}

Géographie critique du glanage alimentaire urbain

\section{Flaminia Paddeu}

\section{(2) OpenEdition}

\section{Journals}

Édition électronique

URL : https://journals.openedition.org/echogeo/17788

DOI : $10.4000 /$ echogeo. 17788

ISSN : 1963-1197

Éditeur

Pôle de recherche pour l'organisation et la diffusion de l'information géographique (CNRS UMR 8586)

Référence électronique

Flaminia Paddeu, «Déchets, mauvaises herbes et plantes sauvages », EchoGéo [En ligne], 47 | 2019, mis en ligne le 21 avril 2019, consulté le 10 août 2021. URL : http://journals.openedition.org/echogeo/ 17788 ; DOI : https://doi.org/10.4000/echogeo.17788

Ce document a été généré automatiquement le 10 août 2021.

EchoGéo est mis à disposition selon les termes de la licence Creative Commons Attribution - Pas d'Utilisation Commerciale - Pas de Modification 4.0 International (CC BY-NC-ND) 


\title{
Déchets, mauvaises herbes et plantes sauvages
}

\author{
Géographie critique du glanage alimentaire urbain
}

\section{Flaminia Paddeu}

Note de l'auteure : la version française de cet article a été traduite par nos soins à partir d'une version originale rédigée en anglais. Nous tenons à remercier Antoine Guironnet pour sa relecture attentive des versions antérieures de cet article.

\section{Introduction}

1 Malgré des recherches encore parcellaires, le glanage alimentaire urbain - = c'est-àdire la cueillette et la récupération de déchets alimentaires - est considéré comme un phénomène mondial. Dans les villes des Nords, il est autant pratiqué par les riches et les pauvres, les actifs et les chômeurs, les migrants et les résidents de longue durée, dont les motifs et les pratiques varient dans le temps et dans l'espace. En tant qu'ensemble de pratiques informelles itinérantes visant à collecter des ressources biologiques ainsi que des déchets alimentaires, le glanage alimentaire urbain est pratiqué dans des territoires urbains, suburbains et périurbains, au sein d'espaces communs, publics ou privés. Ces pratiques de glanage, dépourvues la plupart du temps d'échange monétaire et nécessitant des compétences particulières pour localiser, ramasser et parfois transformer et distribuer les ressources, font généralement l'objet de stratégies de dissuasion de la part des gouvernements locaux.

2 Malgré des caractéristiques communes, la cueillette et la récupération de déchets alimentaires concernent des acteurs, des motifs, des espaces et des représentations distincts. D'une part, la cueillette urbaine consiste à récolter ou à ramasser des ressources biologiques brutes (champignons, plantes voire animaux) en milieu urbain, principalement pour la consommation alimentaire ou médicale directe, la décoration, l'artisanat, les cérémonies, le troc ou l'échange marchand (Shackleton et al., 2017). Elle peut concerner des espèces sauvages ou cultivées, dans des espaces plus ou moins appropriés. Si la cueillette est principalement pratiquée en milieu rural et forestier 
(Short Gianotti et Hurley, 2016), elle a récemment pris de l'ampleur dans des métropoles comme Seattle, Baltimore, New York, Philadelphie ou Syracuse (McLain et al., 2014 ; Plieninger et al., 2015 ; Synk et al., 2017), Stockholm, Berlin ou Edimbourg (von Hoffen et Säumel, 2014) ou encore dans des villes finlandaises ou japonaises (Terada et al., 2010; Kangas et Markkanen, 2001). D'autre part, la récupération de déchets alimentaires est la pratique consistant à récupérer les biens mis au rebut sur les trottoirs, dans les rues ou dans les poubelles. Dans les pays des Nords, l'expression «faire les poubelles» (dumpster diving) a été largement appliquée à diverses formes de récupération des déchets alimentaires. En plus des bennes à ordures, les participant.e.s récupèrent souvent sur les marchés, au bord de la route ou sur les tables de restaurants (des restes d'assiette), forment des arrangements avec les employée.es ou les commerçant.e.s et troquent au sein de réseaux locaux (Oakes, 2000 ; Vinegar, Parker et McCourt, 2016). Un certain nombre d'enquêtes portent ainsi sur ces pratiques de récupération de déchets alimentaires dans des villes globales comme New York et Los Angeles (Barnard, 2011 et 2016b; Nguyen, Chen et Mukherjee, 2014), Montréal (Vinegar, Parker et McCourt, 2016) ou Paris (Guien et Ramirez, 2017).

Plusieurs formes de glanage sont disponibles pour celles et ceux qui ne peuvent accéder au système alimentaire conventionnel, ou qui souhaitent le contourner et le compléter. L'état des connaissances sur les modes de glanage alimentaire en milieu urbain et sur leur géographie est cependant limité. Ainsi, l'objectif de cet article est double. Tout d'abord, il s'agit d'effectuer une revue de la littérature sur les multiples formes de glanage alimentaire en milieu urbain dans les métropoles des Nords. Pour ce faire, nous souhaitons privilégier une approche reposant sur un continuum cueilletterécupération plutôt que d'opposer quête de plantes sauvages et récupération des invendus. Cette approche permet de mieux comprendre les arrangements, les négociations, les résistances et les mobilisations qui soutiennent l'ensemble des formes de glanage. Elle permet également d'aller au-delà d'une analyse idiosyncratique de chaque pratique, pour fournir des connaissances théoriques plus générales sur le glanage alimentaire urbain. À partir de cet état de l'art, il s'agit ensuite de proposer un agenda de recherche dans une perspective de géographique critique. Étant donné que nos modes de subsistance sont ancrés dans des conditions de vie et des capitaux économiques, sociaux, culturels, résidentiels et symboliques interdépendants, le glanage nous sert ici d'analyseur autant de formes structurelles de pouvoir, d'exclusion, d'injustice et d'inégalité que d'alternatives possibles. Les perspectives de recherche que nous proposons puisent dans trois corpus : les études sur l'informalité urbaine, les études radicales sur l'alimentation ainsi que l'économie et l'écologie politiques urbaines. D'une part, les pratiques informelles de glanage aident à comprendre non seulement les stratégies de survie alimentaire, mais aussi les tactiques pour contourner les règlements qui rendent le glanage urbain indésirable. D'autre part, réfléchir à un "droit de glanage" élargit le champ d'application de la justice alimentaire pour permettre de repenser nos relations avec les économies alimentaires capitalistes. Enfin, les modes de glanage urbain interrogent l'accès au foncier et à l'espace, les politiques urbaines, ainsi que les transformations urbaines en contexte capitaliste. 


\section{Analyser le glanage alimentaire urbain : une littérature pluridisciplinaire}

4 Dans les travaux d'anthropologie, d'écologie, de géographie, de sociologie et d'urbanisme, le glanage alimentaire urbain est caractérisé en fonction de qui glane et comment, des motifs et des obstacles au glanage, et du contrôle des pratiques de glanage par les gouvernements locaux.

\section{Des pratiques mutuelles au sein de groupes, d'espaces et de temporalités distincts}

5 Les gens cueillent habituellement toute l'année : particulièrement au printemps, en été et en automne, périodes actives de récolte ; mais l'hiver offre encore des possibilités de ramassage (Poe et al. 2013; Synk et al. 2017). Ils et elles récupèrent aussi les déchets alimentaires tout au long l'année, généralement une à quatre fois par semaine, et parfois même quotidiennement (Vinegar, Parker et McCourt, 2016). Leurs excursions fréquentes exigent et favorisent à la fois des connaissances spécifiques ainsi que des relations particulières avec les lieux, les biens et les espèces qui sont collectés. Les glaneur.se.s urbain.e.s possèdent donc souvent des connaissances d'écologie locales sophistiquées, acquises par l'intermédiaire de la famille et d'amis, de formations botaniques, de guides de terrain et de livres (Poe et al., 2013). Celles et ceux qui récupèrent les déchets alimentaires connaissent les horaires de collecte des ordures $\mathrm{du}$ quartier, les routines de mise au rebut des commerces alimentaires et sont capables d'évaluer les meilleurs sites de collecte (Barnard 2011).

6 Les données démographiques dont on dispose pour l'instant sur les cueilleur.se.s urbain.e.s révèlent des profils particulièrement diversifiés en termes d'âge, de sexe, de revenu et d'origine ethnique, depuis des personnes de classe moyenne supérieure ayant un mode de vie alternatif jusqu'aux individus sans domicile qui vivent dans l'insécurité alimentaire (McLain et al., 2014 ; Poe et al., 2013 ; Shackleton et al., 2017 ; Short Gianotti et Hurley, 2016 ; Synk et al., 2017 ; Vinegar Parker et McCourt, 2016). Les cueilleur.se.s comprennent des résidents autochtones et non-autochtones, ainsi que des migrants nationaux et internationaux (Poe et al., 2013). De même, celles et ceux qui récupèrent les déchets alimentaires présentent une diversité de profils: enseignant.e.s dans l'enseignement secondaire, mères célibataires en situation de pauvreté, migrant.e.s récemment arrivé.e.s (Barnard, 2011 et 2016b ; Ramirez, 2016). Dans certains groupes vulnérables, la moitié de celles et ceux qui font les poubelles sont sans-abris (Eikenberry et Smith, 2005). Par-delà ces profils relativement variés, certains gestes, valeurs et représentations sont partagés par l'ensemble des glaneur.se.s, comme la coopération, le don et la réciprocité sociale (Eikenberry et Smith, 2005 ; Poe et al., 2013).

7 Pourtant, cette «culture matérielle partagée » spécifique (Guien et Ramirez, 2017, p. 49) est mise en œuvre selon des modes, des espaces et des temporalités distincts, suggérant des pratiques latentes d'évitement et d'entre-soi. Par exemple, à Montréal, les individus victimes d'insécurité alimentaire qui font les poubelles, comme les sansabris, ont tendance à être plus isolés que celles et ceux bénéficiant d'une sécurité alimentaire, comme les étudiants, qui ont des liens sociaux plus solides (Vinegar, Parker et McCourt, 2016). Les Blancs semblent préférer faire les poubelles de jour, tandis que les non-Blancs font plus souvent les poubelles de nuit, pour éviter d'être vus 
(ibid.). Dans la région du Pacifique Nord-Ouest aux États-Unis, les cueilleur.se.s de différentes origines ne ramassent pas les mêmes espèces: palommier, ronce remarquable et ortie pour les Autochtones; châtaigne, cresson et plantain pour les Asiatiques; amarante et herbes aromatiques pour les Latinos (Poe et al., 2014). Les nouveaux arrivants identifient et apprécient différemment les espèces de leur pays d'origine : alors que les écologistes de Seattle considèrent le sorbier d'Europe comme une espèce invasive, il est très apprécié par les cueilleur.se.s russes pour ses baies.

\section{Un mode de subsistance marginal}

8 Le glanage alimentaire urbain joue un rôle partiel en terme de subsistance, complétant le plus souvent d'autres modes de subsistance courants façonnés par les systèmes alimentaires conventionnels ou alternatifs. À travers l'autosubsistance et l'accès à des aliments culturellement appropriés, le glanage alimentaire urbain peut contribuer à la sécurité et à la souveraineté alimentaires (McLain et al., 2014; Palliwoda, Kowarik et von der Lippe, 2017 ; Poe et al., 2013). Les espèces cueillies représentent ainsi 1 à 47 \% du régime alimentaire des cueilleur.se.s à Baltimore, avec une contribution moyenne de $7 \%$ et une plus grande dépendance à l'égard de la cueillette pour les cueilleur.se.s les plus pauvres (Synk et al., 2017). De nombreuses personnes souffrant d'insécurité alimentaire préfèrent faire les poubelles plutôt que de dépendre des programmes gouvernementaux ou caritatifs, ou du fait de difficultés d'accès aux banques alimentaires ou aux soupes populaires (Eikenberry et Smith, 2005). En effet, les sansabris sont confronté.e.s à des heures d'ouverture limitées, des problèmes de transport ou d'accès à une cuisine, ou ne répondent pas aux critères de qualification. De plus, nombre d'entre eux cherchent à se distinguer de la stigmatisation sociale et du sentiment de dépendance associés à la charité. Pendant les périodes de crise, qu'elles soient liées à des catastrophes naturelles ou des récessions économiques, le glanage alimentaire urbain sert également de stratégie d'adaptation. Pendant le siège de Sarajevo, 91 espèces de plantes sauvages et de champignons ont ainsi été consommées par les habitants piégés dans l'enceinte de la ville (Redzić, 2010). Plus récemment, à Syracuse (New York), un groupe d'immigrants qui avait perdu son potager urbain a pu satisfaire ses besoins alimentaires quelques temps en ramassant les adventices des friches avoisinantes (Shackleton et al. 2017).

Plutôt que de fournir des ressources nutritives substantielles, le glanage alimentaire urbain est plus souvent utilisé pour transformer la composition et la qualité de l'alimentation des individus (McLain et al., 2014 ; Palliwoda, Kowarik et von der Lippe, 2017). Des cueilleur.se.s ont ramassé 486 espèces différentes à Seattle (Poe et al., 2013) et 170 à Baltimore, le plus souvent des fruits, des noix, des baies et des feuilles (Synk et al., 2017). À Montréal, faire les poubelles permet de manger des aliments plus exotiques et plus chers, notamment des fruits et des légumes, de la viande et des produits laitiers (Vinegar, Parker et McCourt, 2016). En revanche, à Minneapolis, les déchets alimentaires les plus récupérés sont les beignets, la pizza et le pain (Eikenberry et Smith, 2005), ce qui suggère que les glaneur.se.s ramassent selon la disponibilité et les habitudes alimentaires propres au quartier. 


\section{De la politique et du plaisir}

10 Le glanage relève aussi bien du geste politique que du plaisir. Afin d'éviter les modes de consommation et de production capitalistes, certaines personnes expérimentent des stratégies pour atténuer leurs besoins ou les satisfaire différemment. De nombreux.ses glaneur.se.s se décrivent comme " alternatifs » ou « engagés " par rapport à leur mode de vie, leur identité, la politique ou l'idéologie (Vinegar, Parker et McCourt, 2016). Avec l'augmentation des déchets commerciaux, les freegans (Barnard, 2011 et 2016b; Edwards et Mercer, 2012 ; Gross, 2009), les jeunes engagé.e.s (Eikenberry et Smith, 2005) ou les «frugaux volontaires» (Guien et Ramirez, 2017) adoptent des pratiques d'anticonsumérisme. Faire les poubelles est au cœur du freeganisme, une idéologie anticapitaliste revendiquée par des individus qui souhaitent réduire les déchets et minimiser leur empreinte écologique. Les freegans pratiquent aussi le squat, le guerilla gardening, la cueillette, le cyclisme, l'auto-stop, le chômage volontaire et le militantisme (Barnard, 2011).

11 Au-delà des motifs politiques, souvent utilisés pour légitimer le glanage, des auteures font référence à un "principe de plaisir " partagé par les glaneur.se.s qui expriment des sentiments d'excitation, d'addiction ou de divertissement (Guien et Ramirez, 2017), et évoquent inversement de la tristesse ou de la persévérance en cas d'arrêt ou d'interdiction (Poe et al., 2013). La joie de chercher, de classer et de distinguer des plantes pourrait être interprétée comme un héritage des « matrices comportementales de l'animal cueilleur que nous fûmes » (Morizot, 2018, p. 106).

\section{Glaner ou ne pas glaner}

Les pratiques de glanage ont été progressivement marginalisées. En ville, le manque de temps dû aux rythmes urbains contemporains (Synk et al., 2017), la séparation entre le travail et les loisirs, et de puissantes habitudes de consommation alimentaire constituent des obstacles au glanage alimentaire. La récupération de déchets alimentaires prend beaucoup de temps et dépend des heures précises de mise au rebut des commerces alimentaires. Certains freegans ont ainsi abandonné ou limité leurs pratiques par manque de temps pour faire les poubelles (Barnard, 2016b).

Les risques sanitaires associés à la cueillette en milieu urbain pourraient aussi en limiter les potentialités. En plus d'une éventuelle mauvaise identification des espèces, les personnes qui consomment des plantes, des baies et des fruits en milieu urbain peuvent être exposées à des résidus chimiques toxiques (von Hoffen et Säumel, 2014 ; Synk et al., 2017; Weeks et al., 2006). Bien que très peu de cas d'intoxications dues à la cueillette en milieu urbain soient signalés dans la littérature (Poe et al., 2013), ceux dus à la récupération des déchets alimentaires semblent plus fréquents (Vinegar, Parker et McCourt, 2016). De fait, la plupart des glaneur.se.s adaptent leurs pratiques pour éviter les risques sanitaires. En été, les gens font plus souvent les poubelles pour éviter les problèmes d'altération des aliments (Vinegar, Parker et McCourt, 2016). Certain.e.s cueilleur.se.s évitent les sites où les sols sont contaminés ou exposés à la pollution atmosphérique, délaissent les plantes de certaines familles et lavent les matières végétales (Poe et al., 2013 ; Synk et al., 2017). D'autres choisissent leurs lieux de cueillette en fonction de la présence de barrières bâties ou végétales, afin de réduire l'exposition aux sources de pollution. 
Plus généralement, des normes sociales négatives et des stigmates associés au fait de faire les poubelles - et plus largement aux déchets - dissuadent les gens de récupérer les déchets alimentaires (Eikenberry et Smith, 2005 ; Vinegar, Parker et McCourt, 2016). La capacité à surmonter cette stigmatisation varie selon le niveau d'acceptation du non-conformisme au sein des cercles sociaux, sans corrélation systématique avec le statut économique. Par conséquent, l'accès à des réseaux sociaux spécifiques est essentiel pour acquérir les connaissances nécessaires et surmonter les obstacles qui empêchent de glaner.

\section{Contrôler le glanage urbain}

15 Les droits de la propriété et les règlements d'urbanisme constituent des obstacles majeurs au glanage dans les espaces urbains (Hurley et al. 2015 ; Shackleton et al. 2017 ; Svizzero 2016). L'interdiction de cueillir des plantes dans les espaces publics tels que les parcs ou les zones protégées, ainsi que les restrictions concernant le fait de faire les poubelles des supermarchés et des propriétés privées, ont contribué à rendre la pratique du glanage alimentaire urbain indésirable et inappropriée dans les villes contemporaines. Dans la plupart des cas, soit les règlements municipaux ne mentionnent pas explicitement le glanage, soit ils énoncent une forme de restriction ou d'interdiction (Shackleton et al., 2017). Par exemple, le code municipal de Seattle interdit de prélever toute espèce végétale des parcs municipaux, notamment pour éviter d'endommager les ressources du parc (McLain et al., 2014). Les contrevenants à cette loi sont passibles d'une amende maximale de $5000 \$$ ou d'une peine d'emprisonnement maximale d'un an ou deux. Les arbres fruitiers sont souvent interdits le long des voiries urbaines, car les fruits risquent de tomber sur le trottoir, salir la chaussée et augmenter le risque de chutes pour les piétons.

Cependant, le droit de glanage est encore reconnu dans certaines législations, comme en France. Selon l'article R26-10 du Code pénal, le glanage est autorisé du lever au coucher du soleil après la récolte normale, y compris lorsqu'il s'agit d'enfreindre la propriété privée. De même, les déchets non ramassés et les artefacts jetés sont considérés comme des Res Derelictae ou Res Nullius, soit des objets abandonnés qui appartiennent au premier qui les ramasse. En France, ce statut permet de légaliser le fait de faire les poubelles ou de récupérer des objets abandonnés, sous réserve du respect de certaines conditions, comme ne pas disperser les déchets sur la voie publique ou ne pas enfreindre la propriété privée.

17 En parallèle, certains gouvernements municipaux commencent progressivement à soutenir les pratiques de glanage, souvent par le biais de partenariats avec des groupes de la société civile. En France, le Parlement a voté en 2016 une loi contre le gaspillage alimentaire interdisant aux supermarchés de jeter les invendus ou de les rendre impropres à la consommation. À Seattle, la cueillette sur des arbres fruitiers fait son apparition dans les parcs urbains, marquant un changement dans les politiques de gestion des forêts urbaines (McLain et al., 2012). Certaines autorités municipales s'engagent également dans la lutte contre les déchets alimentaires, par exemple à Paris avec le Plan de lutte contre le gaspillage alimentaire 2015-2020 et le Plan Compost parisien 2016-2020. Néanmoins, les types de pratiques qui sont encouragées révèlent un traitement inégal des diverses formes de glanage. Les municipalités utilisent souvent l'interdiction de faire les poubelles comme une législation anti-pauvre. En revanche, la 
cueillette sur les arbres fruitiers ou la collecte d'invendus alimentaires pour des organisations caritatives sont soutenues par les municipalités et présentées comme des pratiques « innovantes » ou « exemplaires ». Faire les poubelles ou glaner de manière informelle demeurent ainsi stigmatisés et découragés, surtout lorsqu'ils sont effectués par et avec des groupes marginalisés tels que les sans-abris, les Roms ou les migrants.

\section{Vers une géographie critique du glanage alimentaire urbain : perspectives de recherche}

18 La mobilisation de trois corpus de géographie critique permet de formuler un agenda de recherche sur le glanage alimentaire urbain. Le recours à la notion d'informalité urbaine pour analyser les pratiques de glanage permet de comprendre pourquoi ces dernières ont longtemps été considérées par les autorités municipales comme indésirables, et comment les pratiques réelles s'articulent avec les règlements et les contournent. Considérer un « droit de de glanage » élargit la portée des théories de la justice alimentaire, en offrant des opportunités pour saisir les relations ambivalentes entre les économies alimentaires capitalistes et les pratiques de glanage. Enfin, l'analyse des «modes de glanage » urbains (Shackleton et al., 2017) aborde la question de l'accès au foncier et à l'espace, des politiques urbaines ainsi que des transformations urbaines en contexte capitaliste.

\section{Rendre les pratiques informelles de glanage indésirables}

19 Glaner de la nourriture - en cueillant dans des lieux non autorisés, en récupérant des aliments laissés à l'abandon et en les vendant parfois - est l'une des manifestations de l'informalité urbaine. L'approche des pratiques de glanage comme une "variété d'arrangements, [qui] concerne aussi bien les citadins qui disposent de peu de ressources et d'accès aux sphères dominantes, que les intermédiaires et les élites " (Jacquot, Sierra et Tadié, 2016, p. 2) permettrait d'en renouveler l'analyse. Comment et où se déroulent les pratiques non officielles de glanage alimentaire urbain? Comment sont-elles prises en compte et réglementées par les pouvoirs publics?

\section{Espaces marginaux et économie du glanage}

Des études plus approfondies sur le glanage alimentaire urbain pourraient contribuer à une meilleure compréhension des espaces et de l'économie de l'informalité urbaine. En tant que catégorisation spatiale, l'informalité est habituellement considérée comme territorialisée à l'intérieur des bidonvilles, dans les marges légales, politiques, économiques, sociales et environnementales de la ville (McFarlane, 2012). Dans les villes des Nords, la spatialité du glanage alimentaire s'ancre dans ces marges : terrains vagues, interstices urbains, zones d'habitats temporaires ou nomades, décharges ou périphéries. Le glanage urbain questionne ainsi le rôle de l'espace dans le soutien des pratiques informelles. Les espaces et les modes de glanage varient en fonction de l'écologie, de l'urbanisation, des réglementations ainsi que des cultures, des goûts, de la saisonnalité ou des capacités de mobilité. Comme les pratiques de glanage sont particulièrement mobiles et labiles, elles dessinent des territoires mouvants, sans limites et en constante évolution. Inversement, ces dernières contribuent à façonner, 
reconfigurer ou transformer les espaces urbains. Cueillir ou stocker de la nourriture peut animer un terrain vague, tandis que glaner des déchets alimentaires peut redonner de la valeur à une benne à ordures.

En tant que forme organisationnelle, l'informalité désigne aussi un travail considéré comme non organisé et non réglementé, alors qu'en réalité ce travail est souvent très organisé et discipliné (McFarlane, 2012). Afin de contourner les systèmes alimentaires conventionnels, la culture, la vente et l'acquisition de nourriture dans les villes se font parfois de façon informelle ou illégale, notamment via la mise en culture d'espaces squattés, le commerce non autorisé de fruits et légumes provenant de jardins familiaux, la vente à la sauvette, le ramassage des invendus sur les marchés de plein-vent, la cueillette dans les parcs publics ou le vol à l'étalage. Le glanage contribue ainsi à l'économie domestique par des échanges informels. On sait peu de choses sur cette économie informelle du glanage alimentaire, mais certaines études de cas montrent qu'elle est très organisée et hiérarchisée au sein des réseaux migratoires, et génère des revenus importants (Hochedez, 2018), tandis que d'autres cas attestent de structures économiques moins organisées (Poe et al., 2013 ; Vinegar, Parker et McCourt, 2016).

\section{Réguler et tolérer le glanage alimentaire urbain}

Les règlements municipaux interdisent souvent la cueillette et la récupération des déchets alimentaires (McLain et al., 2014), rappelant que la distinction entre le formel et l'informel se matérialise souvent comme un outil de gouvernabilité. En effet, les distinctions entre le formel et l'informel sont utilisées par les États comme des dispositifs organisationnels qui permettent ou interdisent des domaines et des formes d'action particuliers (McFarlane, 2012). Bien qu'il soit reconnu dans de nombreux pays, le droit de glanage est flou et limité par les droits d'enclosure des propriétaires privés ou des autorités publiques. Dans de nombreux pays, franchir les clôtures d'un supermarché pour faire les poubelles peut conduire devant la justice pour violation de la propriété privée. Les municipalités peuvent également interdire temporairement le glanage en adoptant des décrets municipaux. Dans les supermarchés, verser des substances toxiques sur les invendus ou protéger les poubelles afin de décourager le glanage est devenu une pratique courante.

Néanmoins, les réglementations sont souvent floues, litigieuses et enfreintes dans les faits. Les règles en vigueur sont sujettes à des interprétations ambiguës ou sont rarement appliquées, ce qui révèle une forme de tolérance à l'égard de ces pratiques, et parfois leur reconnaissance juridique. Par exemple, si de nombreuses règles institutionnelles interdisent la cueillette de plantes sauvages dans les espaces publics, certains gestionnaires de parcs reconnaissent les bienfaits de la sensibilisation à l'environnement et des connaissances écologiques qui découlent de la cueillette. Dans certains cas, les autorités créent mêmes des zones de glanage ad hoc, institutionnalisant les pratiques informelles (McLain et al., 2014).

En retour, les glaneur.se.s déploient des stratégies de négociation, des arrangements, des formes de résistances et des mobilisations pour contourner, lutter contre ou interagir avec les règlements (Jacquot, Sierra et Tadié, 2016). Dans les parcs urbains, la cueillette se pratique de manière discrète et grâce à des ententes tacites avec les gestionnaires (Poe et al., 2013; Short Gianotti et Hurley ,2016). À Paris, un groupe d'éboueurs municipaux tamoul permet à certaines personnes de faire les poubelles en échange de leur aide pour le déplacement des bennes roulantes et le compactage des 
boîtes en carton (Ramirez, 2016). Pourtant, ces arrangements sont intrinsèquement transitoires et sujets à des capacités inégales de mobilisation des ressources et des capitaux. Alors que les communautés freegan s'engagent souvent dans des stratégies de «retournement du stigmate» associé au déchet alimentaire (Nguyen, Chen et Mukherjee, 2014), d'autres communautés comme les sans-abris, les biffins ou les Roms sont plus souvent victimes d'intolérance et continuent à porter le stigmate associé à la récupération. Dans les terrains vagues ou les forêts urbaines, les individus qui cueillent comme pratique de loisir et qui font face à des formes de tolérance, disputent l'usage des lieux aux communautés marginalisées qui utilisent les mêmes espaces, comme les sans-abris et les prostitué.e.s.

\section{L'informalité comme valeur négociable}

25 L'informel est une "valeur négociable » (Alsayyad et Roy, 2006; McFarlane, 2012) aux frontières mouvantes, au sens où les relations entre l'informel et le formel sont négociables et modifiables. En tant que pratiques, l'informel et le formel ne sont pas antinomiques : elles existent comme une sorte de " maillage » ou d'" enchevêtrement » qui se délimitent mutuellement (McFarlane, 2012). Ainsi, la superposition historique de droits de glanage contradictoires dans le droit français indique que la frontière est mince entre le glanage comme collecte et comme vol. En effet, les pratiques de récupération des déchets sont souvent considérées comme menaçant les droits de propriété, et donc assimilées au vol (voir Thomas, 2010) ou à l'intrusion. Cette situation juridique floue met en évidence le caractère historique et politique de la distinction entre formel et informel. En retour, cela signifie que le caractère informel du glanage doit être compris comme une production de l'État lui-même plutôt que comme un état préexistant et indépendant faisant par la suite l'objet d'une réglementation étatique (Roy, 2005).

26 Ainsi la réglementation étatique en matière de glanage révèle et alimente des normes et des stigmates associés à la conservation et à la gestion de l'environnement, ainsi qu'aux droits de propriété. Pour être mieux comprises, les pratiques informelles de glanage alimentaire doivent être encastrées dans les systèmes sociaux et les valeurs qui les structurent: cueillir dans les forêts urbaines a été largement dévalorisé par les paradigmes hygiénistes, récréatifs et conservationnistes, perpétuant l'idée que les villes ne contribuent plus à des modes de subsistance reposant sur des ressources naturelles de proximité (Hurley et Halfacre, 2011). De même, les médias mainstream et les discours politiques opposent souvent les personnes défavorisées qui récupèrent pour des motifs économiques de survie aux personnes plus favorisées qui réutilisent pour des motifs éthiques, politiques ou culturels, comme si les ménages précaires agissaient toujours par nécessité et jamais par choix politique (Guien et Ramirez, 2017).

\section{Glaner la justice alimentaire en ville}

Les travaux sur la cueillette et la récupération des déchets alimentaires abordent des questions telles que l'insécurité (voir Synk et al., 2017) et l'hygiène alimentaires (von Hoffen et Säumel ,2014; Weeks et al., 2006), mais incluent rarement une perspective de justice alimentaire. De même, la littérature sur la justice alimentaire s'est concentrée sur l'agriculture urbaine et les systèmes alimentaires alternatifs (Horst, McClintock et Hoey, 2017), incluant rarement le glanage parmi les ressources, les systèmes et les 
pouvoirs alimentaires alternatifs. La justice alimentaire est considérée comme une proposition radicale croisant la classe, la race, la culture et le genre pour s'attaquer aux inégalités à tous les niveaux du système alimentaire (Holt-Giménez et Wang, 2011). Cette approche considère l'accès à une alimentation saine et l'existence de voies alternatives au régime alimentaire entrepreneurial dominant (Holt-Giménez et Shattuck, 2011) dans un contexte plus large de luttes pour la justice sociale, raciale et environnementale face aux inégalités structurelles (Alkon et Agyeman, 2011). Contrairement à une focalisation plus étroite sur la sécurité alimentaire, la recherche sur la justice et la souveraineté alimentaires examine les dimensions politicoéconomiques du contrôle des ressources alimentaires. Partant, en quoi la revendication d'un «droit au glanage» remet-elle en question la façon dont la production, la distribution et la consommation des aliments reproduisent-elles les inégalités raciales et économiques? De quelles manières le glanage alimentaire urbain participe-t-il à l'exploration des possibilités de relations alternatives aux économies alimentaires capitalistes?

\section{Recouvrir le droit au glanage}

La justice alimentaire reconnaît le droit des populations locales à regagner le contrôle de leurs propres systèmes alimentaires culturels, y compris en termes d'accès aux ressources et aux processus décisionnels affectant les espaces concernés. Ce n'est que récemment que certains ont commencé à étendre ces droits aux systèmes et espaces alimentaires sauvages et locaux (Poe et al., 2013). En assurant la diversité alimentaire par le biais des plantes sauvages, la cueillette urbaine contribue à soutenir les identités culturelles et les relations sociales des populations urbaines, y compris celles des peuples autochtones, des premiers colons et des migrant.e.s récent.e.s. À Portland, par exemple, les organisations locales défendent le droit aux "aliments premiers » (first food) et à la cueillette des racines et des baies que les peuples premiers avaient l'habitude de manger. Les Native Gathering Gardens du parc Thomas Cully abritent par exemple des plantes importantes pour les peuples autochtones de Portland, ainsi qu'une zone réservée à la cueillette de fruits comestibles. Dans les Parcs états-uniens de la côte nord-ouest du Pacifique, des droits de cueillette spécifiques ont été accordés aux autochtones. Dans un contexte postcolonial, la revendication d'un droit à la cueillette des plantes sauvage repose donc sur la reconnaissance des traumatismes sociaux historiques et collectifs et sur l'élimination des inégalités persistantes de race, de sexe, de religion, de citoyenneté et de classe (Slocum, Cadieux et Blumberg, 2016).

Les travaux sur la justice alimentaire examinent également les dimensions politicoéconomiques du contrôle des ressources alimentaires en regardant la façon dont les systèmes alimentaires reproduisent les inégalités raciales et économiques. Cependant, on ne sait toujours pas dans quelle mesure le glanage alimentaire urbain permettrait de lutter contre le racisme structurel et les inégalités de classe. La race a rarement été incluse dans les réflexions sur le glanage alimentaire urbain, bien que le freeganisme ait été critiqué comme un mouvement caractérisé par le "privilège blanc " (Barnard, 2016b), en écho aux débats sur la blanchitude (whiteness), l'« aveuglement racial » (color blindness) et l'entre-soi dans les mouvements alimentaires alternatifs (Guthman, 2008). Si celles et ceux qui récupèrent les déchets alimentaires reconnaissent les relations de pouvoir comme une première étape vers le démantèlement des privilèges, il reste à savoir comment ils s'y attaquent, car le glanage n'est pas nécessairement structuré 
comme un mouvement social collectif. Redécouvrir la comestibilité des mauvaises herbes ou réduire la production de déchets s'accompagnent aussi de formes variées de reproduction des relations de pouvoir.

\section{Sous l'emprise des économies alimentaires capitalistes}

Les travaux sur la justice alimentaire explorent également les possibilités d'autonomie productive des communautés locales indépendantes par rapport aux économies alimentaires capitalistes (Alkon et Agyeman, 2011). Pour les glaneur.se.s qui se revendiquent $\mathrm{du}$ " locavorisme », réduire le gaspillage alimentaire et les « kilomètres alimentaires " revient à manger à partir de sources ultra-locales, par exemple en cueillant des plantes sauvages dans la friche à côté de chez soi ou en faisant les poubelles dans son quartier. Dans cette perspective, le glanage alimentaire urbain permettrait à des individus ou à des groupes sociaux de faire valoir leurs droits à des activités économiques et des modes de subsistance impliquant des échanges noncapitalistes (Poe et al. 2013). Il offrirait ainsi des opportunités pour contrer les tendances expansionnistes du capitalisme et créerait une capacité traverser les crises grâce à des filets de sécurité (Edwards et Mercer, 2012 ; Redzić, 2010).

Pour autant, le glanage se comprend mieux comme une pratique "péricapitaliste ", c'est-à-dire à la fois à l'intérieur et à l'extérieur du capitalisme (Tsing, 2017), que comme une pratique totalement indépendante du capitalisme. Les ressources collectées s'articulent avec des économies et des formes de vie dominées par le capitalisme, les glaneur.se.s continuant le plus souvent à faire une partie de leurs courses au supermarché ou à revendre les ressources collectées dans des circuits marchands, in fine réincorporés dans la circulation des capitaux. Le freeganisme a notamment été critiqué pour son enchevêtrement avec l'économie capitaliste, puisque se nourrir des déchets alimentaires est un moyen supplémentaire d'absorber les surplus du système alimentaire conventionnel capitaliste. Autrement dit, en faisant les poubelles, le freeganisme a tendance à célébrer le gaspillage plutôt qu'à le remettre en question. Paradoxalement, le freeganisme existe le plus souvent dans des villes globales au cœur du capitalisme urbain : à New York, même si les freegans s'opposent au principe du loyer et du salariat, presque tous payent un loyer et gagnent un salaire, se sentant finalement obligés d'accepter ces conditions comme une "réalité désagréable " (Barnard, 2016b) inhérente aux villes globales.

\section{Décrypter les transformations urbaines à travers les « modes de glanage »}

Dans les villes, l'étalement, la privatisation et la perte des communs mettent en péril le glanage alimentaire. Que révèle l'exploration des itinéraires et des habitudes de glanage alimentaire, c'est-à-dire des « modes de glanage » (Shackleton et al., 2017), sur les transformations urbaines? Comment ces modes et leurs reconfigurations peuventils aider à décrypter des dynamiques urbaines plus larges, encastrées dans des alliances de pouvoir, des régimes urbains et des modalités de propriété foncière? 


\section{Urbanisation, densification, gentrification et abandon}

33

l'urbanisation, la densification, la gentrification et l'abandon permet de comprendre la manière dont différentes catégories de personnes ont accès aux pratiques de glanage (Hurley et al., 2015; Shackleton et al., 2017). Ces transformations reconfigurent l'emplacement, la taille, la distribution, la nature, la gestion et l'accès aux territoires et aux espaces verts propices au glanage. Il en résulte souvent une perte d'accès pour les glaneur.se.s, ainsi qu'un changement potentiel dans la diversité des espèces, même si les parcelles restantes offrent encore des possibilités.

processus de densification retirent, fragmentent et détériorent les lieux propices a la cueillette. L'étalement urbain prend une forme parcellaire, qui résulte de la combinaison des politiques de zonage, de conservation des terres et de regroupement. La dégradation écologique des habitats due à l'expansion urbaine se manifeste souvent par l'augmentation des eaux usées dans les lacs, la conversion des zones humides, des forêts et des champs en logements, et la destruction des arbres d'alignement pour élargir les routes. Par conséquent, les cueilleur.se.s à la recherche d'une quantité diversifiée et substantielle de ressources peuvent avoir besoin de traverser des territoires plus vastes (Hurley et al., 2015 ; Synk et al., 2017). De même, l'urbanisation transforme les espaces verts à l'intérieur des limites de la ville, compromettant ainsi l'accès des glaneur.se.s aux ressources naturelles et pratiques de subsistance. En particulier dans les quartiers en cours de gentrification, des conflits sont susceptibles d'advenir entre anciens et nouveaux résidents, dont les modes d'interaction avec les ressources naturelles et les paysages diffèrent.

La vacance foncière est au contraire considérée comme une source d'opportunités pour le glanage dans les zones urbaines, sous forme de terrains vagues, friches et zones non bâties. Dans presque toutes les métropoles des Nords, et plus particulièrement dans les villes en décroissance, les marchés fonciers et les politiques urbaines produisent de la vacance foncière, offrant des opportunités temporaires de colonisation des zones abandonnées par les espèces spontanées et aux cueilleur.se.s d'accéder aux espèces comestibles (Hurley et al., 2015). Par exemple, à Détroit, où la désindustrialisation et la fuite des capitaux ont provoqué une vacance foncière massive, des pratiques de cueillette des plantes sauvages, de glanage des arbres fruitiers, de braconnage ainsi que de récupération de métaux sont réapparues (Millington, 2013; Paddeu, 2016). Le glanage alimentaire urbain pourrait donc être considéré comme un ensemble de pratiques certes marginales mais résilientes dans un contexte d'austérité.

\section{Privatisation, perte des communs et droits fonciers}

L'accès à la terre et aux habitats écologiques constitue un enjeu fondamental pour le glanage. Ainsi, les changements dans les modes de propriété, le régime foncier et la gestion des terres peuvent entraver l'accès aux principales espèces végétales et à la poursuite de la cueillette en milieu urbain (Shackleton et al., 2017). Alors que la cueillette était associée aux régimes de propriété commune dans le monde prémoderne (Svizzero 2016), ces régimes sont devenus rares dans le monde capitaliste, concurrencés par la privatisation. Les droits exclusifs de propriété ont été généralisés, en particulier dans les pays des Nords. Ce changement se traduit par la mise en place de clôtures, de vigiles et de patrouilles régulières, ainsi que par des changements dans la

EchoGéo, 47 | 2019 
conception des aménagements paysagers (Hurley et al., 2015). La reconfiguration des espaces oblige les cueilleur.se.s à s'adapter, à innover et à mieux connaître les sites, les espèces et les usages alternatifs. Néanmoins, les cueilleur.se.s sont inégalement affecté.e.s par les transformations urbaines et foncières. Les communs peuvent être cruciaux pour les populations vulnérables résidant dans des territoires en marge, fortement dépendants de la collecte de ressources naturelles, tandis que les résidents de longue date qui ont établi des réseaux et des droits d'accès, peuvent investir davantage dans l'agriculture urbaine et les espaces privés (Shackleton et al., 2017).

Les pratiques de glanage ouvrent ainsi une réflexion sur les droits fonciers et la justice foncière pour les glaneur.se.s. Selon Holt-Giménez (2017), transformer le système alimentaire sans changer les modes d'accès à la terre, le régime foncier et l'usage du sol est non seulement improbable, mais impossible, car la transformation du système alimentaire exige de changer la politique foncière. Repenser l'accès au foncier en ville exige donc de penser au-delà des questions fondamentales de disponibilité et de répartition spatiale des sites potentiels de glanage, afin d'engager des débats politiques plus profonds sur la race, l'espace et la justice qui façonnent les relations de propriété, et de faire face aux inégalités et aux systèmes d'oppression qui ont depuis longtemps imposé ces relations (Safransky, 2017).

\section{Tenir compte des écologies politiques urbaines}

Comment la répartition des espaces de nature en ville affecte-t-elle potentiellement les cueilleur.se.s, quels espaces deviennent disponibles et comment naviguent-ils et elles dans les relations de pouvoir avec les gestionnaires et les propriétaires fonciers (voir Byrne et Wolch, 2009; Heynen, 2003)? L'écologie politique urbaine constitue une approche permettant de comprendre comment la production d'espaces verts affecte les cueilleur.se.s, en changeant les pratiques matérielles et institutionnelles. De plus, elle permet d'étudier les changements de perception sur les usages "appropriés » des espaces et des espèces végétales, $\mathrm{y}$ compris les stratégies de gestion qui donnent généralement priorité à l'esthétique et aux loisirs (Short Gianotti et Hurley, 2016 ; McLain et al., 2014). Généralement, la plupart des programmes de conservation urbaine interdisent les interactions directes entre les humains, la flore et la faune (Hurley et Halfacre, 2011). En même temps, on constate un regain d'intérêt pour le rôle de la production alimentaire dans certains parcs, notamment par la création de vergers et de paysages agroécologiques dans les parcs urbains et la plantation de cultures vivrières dans les servitudes de voirie (Blanc et Paddeu, 2018 ; McLain et al., 2012). Cependant, certains de ces programmes semblent maintenir un système à deux vitesses dans lequel seul un nombre limité d'espèces plantées à dessein et d'espaces délibérément entretenus sont reconnus, alors que de nombreuses autres espèces, zones et pratiques ne le sont pas.

La diversité biologique et l'accès des cueilleur.se.s aux espèces valorisées sont déterminés par les modèles écologiques, la migration des espèces humaines et nonhumaines et la gestion de l'environnement. S'intéresser aux « modes de glanage » peut nous renseigner sur les caractéristiques écologiques ainsi que sur leurs reconfigurations par le biais de migrations, d'assemblages et d'hybridations. Les zones urbaines peuvent offrir des opportunités grâce à la présence d'« espèces synurbiques ", c'est-à-dire des espèces qui sont plus fréquemment associées aux zones urbaines qu'aux autres écosystèmes (Francis et Chadwick, 2012). La cueillette en milieu urbain 
contribue à faire reconnaître que les espèces dites « invasives " peuvent procurer des avantages écologiques et sociaux. Si la perte de biodiversité due à des pratiques de cueillette potentiellement dommageables est souvent évoquée comme un risque (Shackleton et al. 2017), les exemples sont rares. En revanche, la plupart des cueilleur.se.s traitent et cueillent les plantes de façon à assurer leur vigueur et leur abondance, ainsi qu'à préserver les ressources pour les autres utilisateurs (Poe et al. 2013). Des chercheurs japonais ont même documenté le rôle positif de la collecte régulière de bois de chauffe et de racines comestibles dans la création d'habitats pour les espèces sauvages menacées (Kobori et Primack, 2003), ce qui suggère des relations diplomatiques possibles avec les vivants (Morizot, 2016).

Les déchets alimentaires et les plantes sauvages sont de plus en plus envisagés dans un contexte relationnel plus qu'humain. Comprendre le glanage alimentaire urbain à travers les "écologies relationnelles d'appartenance" (Poe et al., 2013) ouvre des possibilités imaginatives, voire radicales, pour vivre "dans les ruines du capitalisme » (Tsing, 2017). Le glanage alimentaire urbain advient dans des zones boisées préservées de l'urbanisation comme dans des paysages suburbains typiques, dans des plantations ornementales à proximité de projets d'aménagement et de centres commerciaux, sur des terre-pleins de stationnements ou entre les autoroutes. Des alliances surprenantes surgissent au sein de ce que Tsing appelle les « communs latents » (ibid.), ouverts aux non-humains et aux enchevêtrements mutuels, inégaux et sélectifs, interstitiels et résistants à l'institutionnalisation, troubles et non contrôlés. Par exemple, face à l'évolution de la croissance urbaine en Caroline du Sud, certain.e.s glaneur.se.s en situation de précarité ont commencé à établir des relations de coopération avec des nouveaux arrivants suburbains aisés vivant dans des résidences sécurisées, afin de faciliter leur accès à l'avoine odorante (sweetgrass) dont ils et elles ont besoin pour faire de la vannerie (Hurley et al., 2015). Ainsi, ces glaneur.se.s ont créé de nouvelles relations avec les paysages urbains ainsi que de nouvelles formes de "communs latents", dans des aménagements paysagers suburbains coûteux, à travers des alliances inattendues et ambivalentes, caractérisées par un accès précaire aux ressources et dans des conditions inégales.

\section{Conclusion}

41 Cet article visait à définir le programme d'une géographie critique du glanage alimentaire urbain. Il s'est appuyé sur un état de l'art pluridisciplinaire sur la cueillette et la récupération de déchets alimentaires afin de proposer un agenda de recherche sur le glanage alimentaire urbain. S'appuyant sur trois corpus de géographique critique, à savoir les études sur l'informalité urbaine, les études radicales sur l'alimentation et l'économie et l'écologie politiques urbaines, cet article explique comment l'étude du glanage alimentaire urbain permet l'analyse des formes structurelles de pouvoir, d'exclusion, d'injustice, d'inégalité comme celle des alternatives dans les espaces urbains du capitalisme.

Ce qui ressort de cet état de l'art est que le glanage alimentaire urbain semble favoriser des pratiques et une culture mutuelles entre différents groupes sociaux, bien qu'elles adviennent dans des espaces et selon des temporalités distincts, suggérant des pratiques latentes d'évitement et d'«entre-soi». Plusieurs obstacles tels que les habitudes de consommation alimentaire, le manque de temps, la sécurité sanitaire et la 
stigmatisation rendent particulièrement difficile l'intégration du glanage alimentaire dans la vie urbaine, qui reste inégalement accessible selon les groupes sociaux. Si quelques gouvernements municipaux ont commencé à soutenir les pratiques de cueillette, l'enclosure des terres et les règlements anti-glanage contribuent à faire du glanage alimentaire une pratique indésirable dans les villes contemporaines. Surtout, le glanage alimentaire véhicule des représentations divergentes : alors que le fait de faire les poubelles continue de porter le stigmate associé aux déchets, la cueillette, associée à la revitalisation des relations avec la faune et la flore urbaine, a récemment pris de l'ampleur. Alors que les pratiques de glanage alimentaire urbain évoluent rapidement, il est important de demeurer attentif aux problèmes éventuels de partage conflictuel des espaces et des ressources entre des individus aux capitaux inégaux, d'autant plus que la cueillette urbaine attire de plus en plus les citadins de classe moyenne et supérieure.

À partir de ces analyses, la mise en regard de différents corpus de travaux de recherche permet de proposer un agenda de recherche sur le glanage alimentaire urbain. Premièrement, les études sur l'informalité urbaine montrent que même si les réglementations considèrent la plupart du temps le glanage alimentaire urbain comme indésirable, les pratiques de tolérance dans les faits révèlent un système à deux vitesses qui repose sur un traitement inégal des groupes sociaux qui glanent. Étant donné que l'informalité urbaine est une valeur négociable produite par l'État (Roy 2005), les réglementations étatiques révèlent et alimentent les normes et stigmates associés au glanage en termes de race, de classe sociale, de conservation et de gestion environnementales et de droits de propriété. Deuxièmement, dans une perspective postcoloniale, les études radicales sur l'alimentation invitent à reconnaître les traumatismes historiques et sociaux collectifs et à mettre fin aux inégalités persistantes de race, de sexe, de religion, de citoyenneté et de classe pour recouvrir un droit au glanage. Cependant, certains doutes subsistent quant à la capacité des glaneur.se.s à affronter le racisme structurel et les inégalités de classe en particulier, car les cueilleur.se.s et les récupérateur.ice.s reproduisent les relations de pouvoir. Si le glanage alimentaire urbain vise une plus grande autonomie productive, son articulation étroite avec les systèmes alimentaires et les économies capitalistes le rend plus susceptible d'être exploré comme une " pratique péricapitaliste ", à l'intérieur et à l'extérieur du capitalisme (Tsing 2017). Troisièmement, l'examen des «modes de glanage » urbains (Shackleton et al. 2017) permet d'étudier la diminution de l'accès aux opportunités de glanage à cause de la privatisation et la perte des communs, ainsi que des changements des caractéristiques sociodémographiques, des représentations et des paysages. Comme les glaneur.se.s sont inégalement affecté.e.s par ces transformations en fonction des capitaux sociaux et fonciers, cela soulève une question de «justice foncière ", encastrée dans des inégalités et des systèmes d'oppression structurels. Ces systèmes de pouvoir inégaux ouvrent la voie à des alliances inattendues et fondamentalement ambivalentes à travers des « communs latents » qui émergent dans les « ruines du capitalisme » (Tsing, 2017).

L'étude du glanage urbain ouvre d'autres pistes de recherche pluridisciplinaires sur la généalogie des droits de glanage, l'émergence de communs à travers le glanage alimentaire urbain et les "relations diplomatiques avec les vivants» (Morizot ,2016). Elle ouvre aussi la voie à une approche relationnelle du glanage alimentaire urbain, tant dans les Suds que dans les Nords, étant donné que la cueillette est pratiquée à Bangalore ou à Delhi (Gopal et Nagendra, 2014), en Ouganda, au Sénégal (NDao et 
Mbaye, 2017) et en Afrique du Sud, et que la récupération est bien documentée à Calcutta et Faisalabad (Batool et Anjum, 2016). Paradoxalement, le " déchétarisme »a été considéré comme une pratique alternative volontaire dans les villes des Nords alors que la « fouille des poubelles » a été plus souvent associée aux personnes dans le besoin dans les villes des Suds. Une étude comparative plus approfondie entre Suds et Nords, ancrée dans les rapports de domination et de pouvoir postcoloniaux, pourrait ouvrir de nouveaux horizons pour penser le glanage alimentaire urbain.

\section{BIBLIOGRAPHIE}

Alkon A., Agyeman J. (eds), 2011. Cultivating Food Justice: Race, Class, and Sustainability. Cambridge, MA, MIT Press, 408 p.

Alsayyad N., Roy A., 2006. Medieval Modernity: On Citizenship and Urbanism in a Global Era. Space and Polity, vol. 10, $\mathrm{n}^{\circ}$ 1, p. 1-20.

Barnard A., 2011. 'Waving the Banana' at Capitalism: Political Theater and Social Movement Strategy among New York's ‘Freegan’ Dumpster Divers. Ethnography, vol. 12, nº 4, p 419-444.

Barnard A., 2016. Making the City 'Second Nature': Freegan 'Dumpster Divers' and the Materiality of Morality. American Journal of Sociology, vol. 121, n 4, p. 1017-1050.

Batool Z., Anjum F., 2016. A sociological study of trash picker children in Faisalabad City, Punjab, Pakistan. Pakistan Journal Life of Social Sciences, vol. 14, $\mathrm{n}^{\circ} 1, \mathrm{p} .33-37$.

Blanc N., Paddeu F., 2018. L'environnementalisme ordinaire. Transformer l'espace public métropolitain à bas bruit ? EspaceTemps.net [En ligne]. https://www.espacestemps.net/articles/ lenvironnementalisme-ordinaire-transformer-lespace-public-metropolitain-a-bas-bruit/

Byrne J., Wolch J., 2009. Nature, Race, and Parks: Past Research and Future Directions for Geographic Research. Progress in Human Geography, vol. 33, n 6, p. 743-765.

Edwards F., Mercer D., 2012. Food Waste in Australia: The Freegan Response. The Sociological Review, vol. 60, $\mathrm{n}^{\circ}$ 2, p. 174-191.

Eikenberry N., Smith C., 2005. Attitudes, Beliefs, and Prevalence of Dumpster Diving as a Means to Obtain Food by Midwestern, Low-Income, Urban Dwellers. Agriculture and Human Values, vol. 22, $\mathrm{n}^{\circ} 2$, p. 187-202.

Francis R., Chadwick M., 2012. What Makes a Species Synurbic? Applied Geography, vol. 32, n² p. 514-521.

Gopal D., Nagendra H., 2014. Vegetation in Bangalore's Slums: Boosting Livelihoods, Well-Being and Social Capital. Sustainability, vol. 6, n 5, p. 2459-2473.

Gross J., 2009. Capitalism and Its Discontents: Back-to-the-Lander and Freegan Foodways in Rural Oregon. Food and Foodways, vol. 17, n 2, p. 57-79.

Guien J., Ramirez V., 2017. Travailler à consommer, Labouring consumption. Terrains \& travaux, $\mathrm{n}^{\circ} 31$, p. 45-62. 
Guthman J., 2008. Bringing Good Food to Others: Investigating the Subjects of Alternative Food Practice. Cultural Geographies, vol. 15, n 4, p. 431-447.

Heynen N., 2003. The Scalar Production of Injustice within the Urban Forest. Antipode, vol. 35, $\mathrm{n}^{\circ} 5$, p. $980-998$.

Hochedez C., 2018. Migrer et cultiver la ville : l'agriculture communautaire à Malmö (Suède). Urbanités [En ligne], $\mathrm{n}^{\circ}$ 10. http://www.revue-urbanites.fr/10-hochedez-malmo/

Hoffen L-P., Säumel I., 2014. Orchards for Edible Cities: Cadmium and Lead Content in Nuts, Berries, Pome and Stone Fruits Harvested within the Inner City Neighbourhoods in Berlin, Germany. Ecotoxicology and Environmental Safety, $n^{\circ} 101$, p. 233-239.

Holt-Giménez E., 2017. A Foodie's Guide to Capitalism: Understanding the Political Economy of What We Eat. Oakland, CA, Monthly Review Press, 256 p.

Holt-Giménez E., Shattuck A., 2011. Food Crises, Food Regimes and Food Movements: Rumblings of Reform or Tides of Transformation? The Journal of Peasant Studies, vol. 38, n 1, p. 109-144.

Holt-Giménez E., Wang Y., 2011. Reform or Transformation? The Pivotal Role of Food Justice in the U.S. Food Movement. Race/Ethnicity: Multidisciplinary Global Contexts, vol. 5, n 1, p. 83-102.

Horst M., McClintock N., Hoey L., 2017. The Intersection of Planning, Urban Agriculture, and Food Justice: A Review of the Literature. Journal of the American Planning Association, vol. 83, $\mathrm{n}^{\circ} 3$, p. 277-295.

Hurley P., Emery M., McLain R., Poe M., Grabbatin B., Goetcheus C., 2015. Whose Urban Forest? The Political Ecology of Foraging Urban Nontimber Forest Products. In Issenhour C., McDonogh G. Checker M., Sustainability in the Global City, Myth and Practice. New York, Cambridge University Press, p. 187-212.

Hurley P., Halfacre A., 2011. “odging Alligators, Rattlesnakes, and Backyard Docks: A Political Ecology of Sweetgrass Basket-Making and Conservation in the South Carolina Lowcountry, USA. GeoJournal, vol. 76, 4, p. 383-399.

Jacquot S., Sierra A., Tadié J., 2016. "Informalité politique, pouvoirs et envers des espaces urbains." L'Espace Politique. Revue en ligne de géographie politique et de géopolitique, n² 29, http:// journals.openedition.org/espacepolitique/3805

Kangas K., Markkanen P., 2001. Factors Affecting Participation in Wild Berry Picking by Rural and Urban Dwellers. Silva Fennica, vol. 35, n 4, p. 487-495.

Kobori H., Primack R., 2003. Participatory Conservation Approaches for Satoyama, the Traditional Forest and Agricultural Landscape of Japan. Ambio, vol. 32, nº 4, p. 307-311.

McFarlane C., 2012. Rethinking Informality: Politics, Crisis, and the City. Planning Theory \& Practice, vol. 13, n 1, p. 89-108.

McLain R., Hurley P., Emery M., Poe M., 2014. Gathering 'Wild' Food in the City: Rethinking the Role of Foraging in Urban Ecosystem Planning and Management. Local Environment, vol. 19, $\mathrm{n}^{\circ} 2$, p. 220-240.

McLain R., Buttolph L., Poe M., MacFarland K., et al. 2012. Gathering in the City: An Annotated Bibliography and Review of the Literature about Human-Plant Interactions in Urban Ecosystems [En ligne]. http://digitalcommons.ursinus.edu/environment_fac/10/

Millington N., 2013. Post-Industrial Imaginaries: Nature, Representation and Ruin in Detroit, Michigan. International Journal of Urban and Regional Research, vol. 37, nº 1, p. 279-296. 
Morizot B., 2016. Les Diplomates, Cohabiter avec les loups sur une nouvelle carte du vivant. Paris, Wildproject, $320 \mathrm{p}$.

Morizot B., 2018. Sur la piste animale. Actes Sud. Paris, Mondes Sauvages, 200 p.

NDao M., MBaye Diouf E., 2017. L'arboriculture fruitière, une menace pour la biodiversité, exemple de la commune de Niaguis (Zinguichor, Sénégal), Liens, vol. 23, n² 2, p. 176-189.

Nguyen H., Chen S., Mukherjee S., 2014. Reverse Stigma in the Freegan Community. Journal of Business Research, vol. 67, nº 9, p. 1877-1884.

Oakes P., 2000. Why Freegan? An Attack on Consumption - In Defense of Donuts [En ligne]. https:// freegan.info/what-is-a-freegan/freegan-philosophy/why-freegan-an-attack-on-consumption-indefense-of-donuts/

Paddeu F., 2016. Les ruines de Détroit, fléaux ou opportunités de la décroissance urbaine ? Vers une éthique politique de la ruine. Frontières [En ligne], vol. 28, n 1. https://id.erudit.org/ iderudit/1038863ar

Palliwoda J., Kowarik I., Lippe M., 2017. Human-Biodiversity Interactions in Urban Parks: The Species Level Matters. Landscape and Urban Planning, $\mathrm{n}^{\circ}$ 157, p. 394-406.

Plieninger T., Bieling C., Fagerholm N., Byg A., et al., 2015. The Role of Cultural Ecosystem Services in Landscape Management and Planning. Current Opinion in Environmental Sustainability, $\mathrm{n}^{\circ} 14$, p. 28-33.

Poe M., Le Compte J., McLain R., Hurley P., 2014. Urban Foraging and the Relational Ecologies of Belonging. Social \& Cultural Geography, vol. 15, n 8, p. 901-919.

Poe M., McClain R., Emery M., Hurley P., 2013. Urban Forest Justice and the Rights to Wild Foods, Medicines, and Materials in the City. Human Ecology, vol. 41, n 3, p. 409-422.

Ramirez V., 2016. De l'art de chambarder la société de consommation. Portrait d'un récupérateur. Techniques \& Culture, $\mathrm{n}^{\circ}$ 65-66, p. 290-293.

Redzić S., 2010. Use of Wild and Semi-Wild Edible Plants in Nutrition and Survival of People in 1430 Days of Siege of Sarajevo during the War in Bosnia and Herzegovina (1992-1995). Collegium Antropologicum, vol. 34, $\mathrm{n}^{\circ}$ 2, p. 551-570.

Roy A., 2005. Urban Informality: Toward an Epistemology of Planning. Journal of the American Planning Association, vol. 71, n², p. 147-158.

Safransky S., 2017. Rethinking Land Struggle in the Postindustrial City. Antipode, vol. 49, $\mathrm{n}^{\circ} 4$, p. $1079-1100$.

Shackleton C., Hurley P., Dahlberg A., Emery M., Nagendra H., 2017. Urban Foraging: A Ubiquitous Human Practice Overlooked by Urban Planners, Policy, and Research. Sustainability, vol. 9, $\mathrm{n}^{\circ} 10$, p. 1884. https://doi.org/10.3390/su9101884

Short Gianotti A., Hurley P., 2016. Gathering Plants and Fungi along the Urban-Rural Gradient: Uncovering Differences in the Attitudes and Practices among Urban, Suburban, and Rural Landowners. Land Use Policy, n 57, p. 555-563.

Slocum R., Cadieux K., Blumberg R., 2016. Solidarité, espace et « race » : vers des géographies de la justice alimentaire." Justice Spatiale Spatial Justice [En ligne], $\mathrm{n}^{\circ}$ 9. https://www.jssj.org/article/ solidarite-espace-et-race-vers-des-geographies-de-la-justice-alimentaire/

Svizzero S., 2016. Foraging Wild Resources: Evolving Goals of an Ubiquitous Human Behavior. Anthropology, vol. 4, $\mathrm{n}^{\circ}$ 1, p. 1-9. 
Synk C., Kim B., Davis C., Harding J., et al., 2017. Gathering Baltimore's Bounty: Characterizing Behaviors, Motivations, and Barriers of Foragers in an Urban Ecosystem. Urban Forestry \& Urban Greening, $\mathrm{n}^{\circ} 28$, p. 97-102.

Terada T., Yokohari M., Bolthouse J., Tanaka N., 2010. Refueling Satoyama Woodland Restoration in Japan: Enhancing Restoration Practice and Experiences through Woodfuel Utilization. Nature and Culture, vol. 5, $n^{\circ} 3$, p. 251-276.

Thomas S., 2010. Do Freegans Commit Theft? Legal Studies, vol. 30, n 1, p. 98-125.

Tsing A., 2017. The Mushroom at the End of the World. Princeton, NJ, Princeton University Press, $352 \mathrm{p}$.

Vinegar R., Parker P., McCourt G., 2016. More than a Response to Food Insecurity: Demographics and Social Networks of Urban Dumpster Divers. Local Environment, vol. 21, n 2, p. 241-253.

Weeks C., Croasdale M., Osborne M., Hewitt L., et al., 2006. Multi-Element Survey of Wild Edible Fungi and Blackberries in the UK. Food Additives \& Contaminants, vol. 23, n 2, p. 140-147.

\section{RÉSUMÉS}

Cet article propose de définir la portée et le programme liminaire d'une géographie critique du glanage alimentaire urbain. Pour cela, nous effectuons une revue de littérature pluridisciplinaire portant sur le glanage d'espèces comestibles sauvages ou cultivées ainsi que de déchets alimentaires, dans le but de développer une analyse théorique du glanage alimentaire dans les villes contemporaines des Nords. Faisant appel à trois corpus de la géographique critique, à savoir les études sur l'informalité urbaine, les études radicales sur l'alimentation, l'économie et l'écologie politiques urbaines, nous soutenons l'idée que l'étude du glanage alimentaire urbain permet d'analyser des formes structurelles de pouvoir, d'exclusion, d'injustice et d'inégalité ainsi que les alternatives dans les espaces du capitalisme.

The aim of this paper is to define the scope and an initial agenda for a critical geography of urban food collecting. It provides an overview of a multidisciplinary literature regarding patterns of foraging and scavenging to develop a theoretical comprehension of urban collecting in contemporary northern cities. Drawing on three bodies of critical geography literature, namely urban informality studies, radical food studies, and urban political economy and ecology, it advocates that investigating urban food collecting provides a tool to analyze structural forms of power, exclusion, injustice and inequality as well as alternative pathways in spaces of advanced capitalism.

\section{INDEX}

Mots-clés : cueillette, géographie critique, glanage alimentaire, récupération, systèmes alimentaires alternatifs

Keywords : alternative food systems, critical geography, foraging, urban food collecting, scavenging

Thèmes : Sur le Champ - Sur le Terrain 


\section{AUTEUR}

\section{FLAMINIA PADDEU}

Flaminia Paddeu, flaminia.paddeu@univ-paris13.fr, est Maître de Conférences en géographie, Université de Paris 13-Villetaneuse, laboratoire PLEIADE - EA 7338. Elle a récemment publié - Blanc N., Paddeu F., 2018. L'environnementalisme ordinaire. Transformer l'espace public métropolitain à bas bruit ?. EspacesTemps.net [En ligne], Travaux. https://www.espacestemps.net/ articles/lenvironnementalisme-ordinaire-transformer-lespace-public-metropolitain-a-bas-bruit/ - Paddeu F., 2017. Sortir du mythe de la panacée. Les ambiguïtés de l'agriculture urbaine à Détroit. Métropolitiques [En ligne]. http://www.metropolitiques.eu/Sortir-du-mythe-de-lapanacee-Les.html

- Paddeu F., 2017. Agir à l'heure de l'anthropocène. Les quatre familles de l'environnementalisme urbain. L'Information géographique, vol. 81, n 3, p. 32-53. 